Article

\title{
Xanthate Based Radical Cascade Toward Multicomponent Formation of Pyrrolopyrimidines
}

\author{
Laurent El Kaïm *, Laurence Grimaud * and Patil Pravin \\ Laboratoire DCSO ENSTA-Polytechnique-CNRS, UMR 7652, Ecole Nationale Supérieure de \\ Techniques Avancées, 32 Bd Victor, 75739 Paris Cedex 15, France \\ * Authors to whom correspondence should be addressed; E-Mails: laurent.elkaim@ensta.fr (L.E.K.); \\ grimaud@dcso.polytechnique.fr (L.G.); Tel.: +33145525537; Fax: +33145528322.
}

Received: 28 September 2011; in revised form: 23 October 2011 / Accepted: 28 October 2011 / Published: 4 November 2011

\begin{abstract}
A short sequential synthesis of pyrrolidino- pyridines and pyrimidines illustrates the potential of combining Ugi-Smiles couplings with radical tin-free processes.

Keywords: Ugi-Smiles coupling; xanthates; multicomponent reactions; radical processes; isocyanides
\end{abstract}

\section{Introduction}

Among multi-component reactions (MCRs), the Ugi reaction has a privileged position due to its broad synthetic significance [1,2]. Its four points of diversity combined with possible further transformations of the resulting adducts have became very popular in heterocyclic synthesis. Indeed, the Ugi reaction has been associated with a wide range of post-condensation reactions in order to form even more complex scaffolds, including cycloadditions [3-9], metal-catalyzed processes [10-15] and cyclocondensations [16-21]. Among this very impressive bibliography, the combination of Ugi or Passerini reactions with radical chemistry has been scarcely documented [22-27]. Among the various radical systems explored in our group, we have mostly studied the potential of xanthate radical transfer resulting in various cyclizations of Ugi xanthate adducts bearing suitable alkene [22], alkyne [25] or aryl [26] moieties. In all these examples, $\alpha$-chloroacetic acid was used as the acidic partner to introduce the xanthate moiety via a nucleophilic substitution. However, these intramolecular post-condensations did not exploit the full potential of the xanthate radical transfer. Indeed, compared 
to tin hydride chemistry, the reversible nature of the addition of radicals onto the thiocarbonyl group is associated with high yielding intermolecular couplings between xanthates and alkenes [28,29]. Herein, we propose to explore this feature and to further increase the molecular diversity of the Ugi step through a xanthate triggered addition/cyclization cascade to form new heterocyclic systems (Scheme 1).

Scheme 1. Synthetic strategy towards pyrrolidino fused systems.

Former strategy

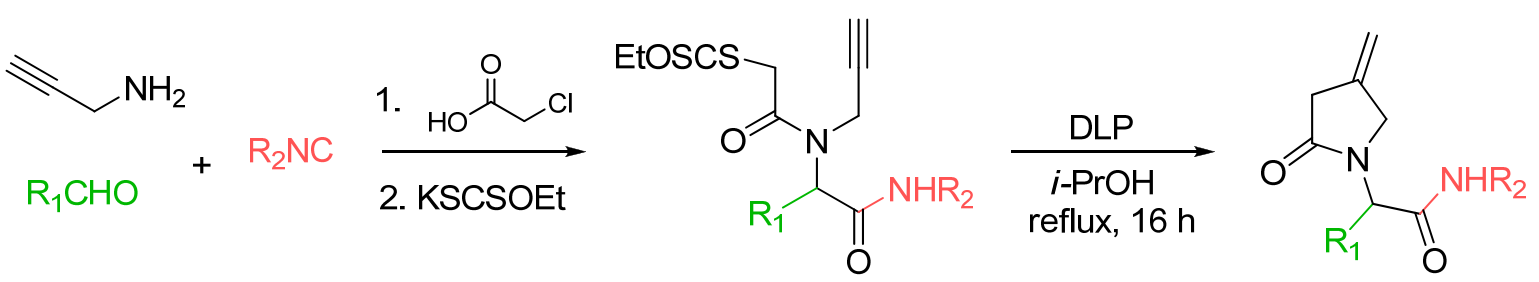

New strategy
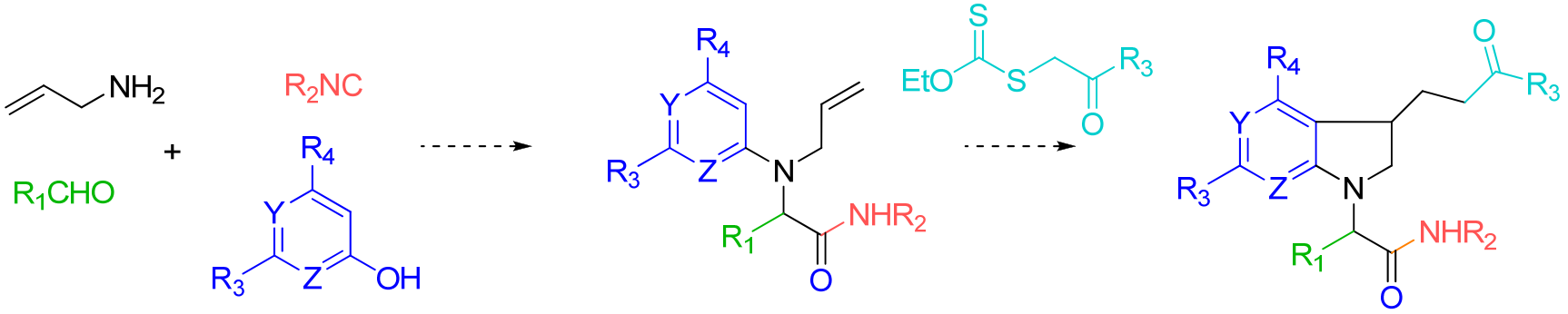

\section{Results and Discussion}

Developing the radical chemistry of xanthates, the Zard group has reported various synthetic approaches towards fused cyclic systems such as indanes, indolines or azaindolines [30-35]. These syntheses feature an intermolecular addition of a xanthate on an alkene followed by an intramolecular trapping with a suitably positioned aryl group. As part of our ongoing interest in Ugi-type reactions, we naturally imagined to synthesize the radical acceptor through a Ugi-Smiles coupling. Indeed, we recently developed the use of electron-deficient phenols as acid surrogates in Ugi reactions to form $\mathrm{N}$-arylamino carboxamides [36,37]. The use of a partner substituted with an alkene, such as allylamine, in this reaction would afford in one step an interesting substrate to test the tandem radical process (Scheme 1). Concerning the phenol, most reported Ugi-Smiles couplings involve a nitro group to perform the Smiles rearrangement. As nitroaryl derivatives are often associated with inhibition of radical chain processes, we prefer the use of hydroxy heterocycles (pyridines, pyrimidines) which also led to efficient multicomponent couplings [38]. $\mathrm{N}$-allylaminopyridines and $\mathrm{N}$-allylaminopyrimidines are known to react with xanthate under radical conditions, however the scope of these reactions seems to be limited by the nucleophilic behavior of the cyclic nitrogen atom. Indeed all reported radical tandem were performed on 2- or 5-halosubstituted heterocycles (chloro and fluoro) in order to lower the reactivity at the nitrogen center (Scheme 2) [31-35]. 
Scheme 2. Selected examples from Zard's group [31,33].<smiles>C=CCN(C)c1cccc(Cl)n1</smiles><smiles>CCCSC(CCC(C)=O)CN(CC(C)(C)C)c1cccc(Cl)c1</smiles><smiles>CC(=O)CCC1CN(C)c2nc(Cl)ccc21</smiles><smiles>C=CCN(C)c1cc(Cl)nc(Cl)n1</smiles><smiles>CN(CCS)c1cc(Cl)nc(Cl)n1</smiles><smiles>CCCCOC(=O)COS(=O)(=O)OCC</smiles><smiles>COC(=O)CCC1CN(C)c2nc(Cl)nc(Cl)c21</smiles>

\subsection{Four-Component Coupling Towards Radical Substrates}

Various Ugi-Smiles adducts were thus prepared starting with allylamine as the amine partner. The latter was coupled with different hydroxy- pyridines and pyrimidines. The MCR coupling was performed under classical conditions using methanol as solvent. The starting radical substrates were obtained in modest to good yields.

Table 1. Preparation of the radical substrates.

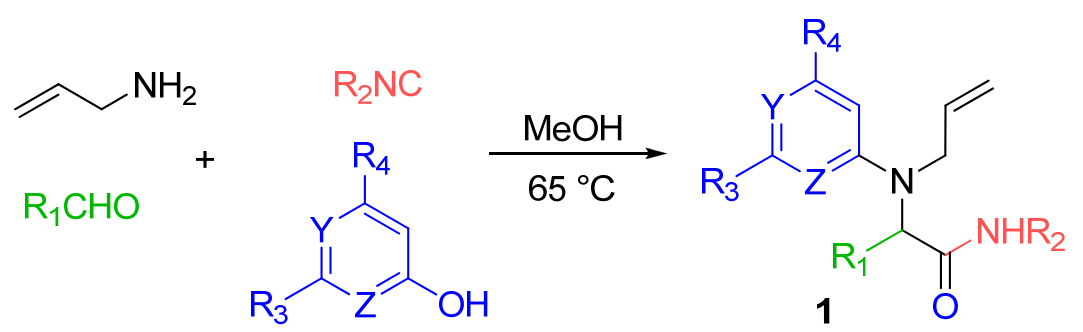

\begin{tabular}{|c|c|c|c|c|c|c|c|}
\hline Entry & $\mathbf{R}_{\mathbf{1}}$ & $\mathbf{R}_{\mathbf{2}}$ & $\mathbf{R}_{\mathbf{3}}$ & $\mathbf{R}_{\mathbf{4}}$ & $\mathbf{Y}$ & $\mathbf{Z}$ & $\begin{array}{c}\text { Product } \\
(\mathbf{\%})\end{array}$ \\
\hline 1 & $\mathrm{Et}$ & $\mathrm{Cy}$ & $\mathrm{Me}$ & $\mathrm{Me}$ & $\mathrm{N}$ & $\mathrm{N}$ & $\mathbf{1 a}(38 \%)$ \\
2 & $i-\mathrm{Bu}$ & $\mathrm{Cy}$ & $\mathrm{H}$ & $\mathrm{H}$ & $\mathrm{N}$ & $\mathrm{CH}$ & $\mathbf{1 b}(69 \%)$ \\
3 & $i-\mathrm{Bu}$ & $t-\mathrm{Bu}$ & $\mathrm{H}$ & $\mathrm{H}$ & $\mathrm{N}$ & $\mathrm{CH}$ & $\mathbf{1 c}(43 \%)$ \\
4 & $\mathrm{Et}$ & $\mathrm{Cy}$ & $\mathrm{Ph}$ & $\mathrm{Me}$ & $\mathrm{N}$ & $\mathrm{N}$ & $\mathbf{1 d}(61 \%)$ \\
5 & $\mathrm{H}$ & $p-\mathrm{MeBn}$ & $\mathrm{Ph}$ & $\mathrm{Me}$ & $\mathrm{N}$ & $\mathrm{N}$ & $\mathbf{1 e}(60 \%)$ \\
6 & $\mathrm{Et}$ & $\mathrm{Cy}$ & $\mathrm{H}$ & $\mathrm{H}$ & $\mathrm{CNO}_{2}$ & $\mathrm{~N}$ & $\mathbf{1 f}(41 \%)$ \\
7 & $i-\mathrm{pr}$ & $p-\mathrm{ClBn}$ & $\mathrm{Me}$ & $\mathrm{Me}$ & $\mathrm{N}$ & $\mathrm{N}$ & $\mathbf{1 g}(68 \%)$ \\
\hline
\end{tabular}

\subsection{Radical Cascade}

In previous studies on xanthate additions on $\mathrm{N}$-allylamino- pyridines and pyrimidines, a first 1,2-addition product on the alkene could be obtained and isolated under heating with substoichiometric amounts of a radical initiator such as dilauroyl peroxide (DLP) in refluxing 1,2-dichloroethane (DCE) or ethyl acetate. Then the intermediate adducts could cyclize under treatment with further DLP or di-tert-butyl peroxide (more than 1 equivalent) at higher temperature allowing cleaner reactions (Scheme 3 ). 
Even if the yields are expected to be lower, we preferred to perform the cascade under a single set of experimental conditions in order to keep the multicomponent sequence as short as possible. When adduct 1a was refluxed in DCE $(0.2 \mathrm{M})$ with DLP added in $15 \mathrm{~mol} \%$ portions every twenty minutes, we could isolate the desired cyclized pyrrolidine 2a as a 9:1 mixture of diastereomers [39] in 48\% isolated yield, after overall addition of 1.5 equivalent of DLP (Scheme 3 ).

Scheme 3. Radical reaction conditions.<smiles>C=CCN(c1cc(C)nc(C)n1)C([14CH3])C(=O)NC1CCCCC1</smiles>

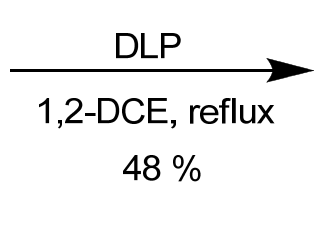<smiles>CCOC(=O)CCC1CN(C(CC)C(=O)NC2CCCCC2)c2nc(C)nc(C)c21</smiles>

This sequence is initiated by decomposition of the xanthate into the electrophilic radical $\mathbf{I}$, which is then trapped by the alkene moiety to give intermediate II. The latter is an electron-rich radical that can react with the starting xanthate to form intermediate IV. The latter being slightly less reactive than the starting xanthate towards radicals issued from DLP, accumulates in the medium and reacts when further amount of DLP is added without much 1 left to enter in the chain process. After addition of the radical onto the aryl ring, the intermediate species III further aromatize through interaction with the DLP (Scheme 4).

Scheme 4. Plausible mechanism.

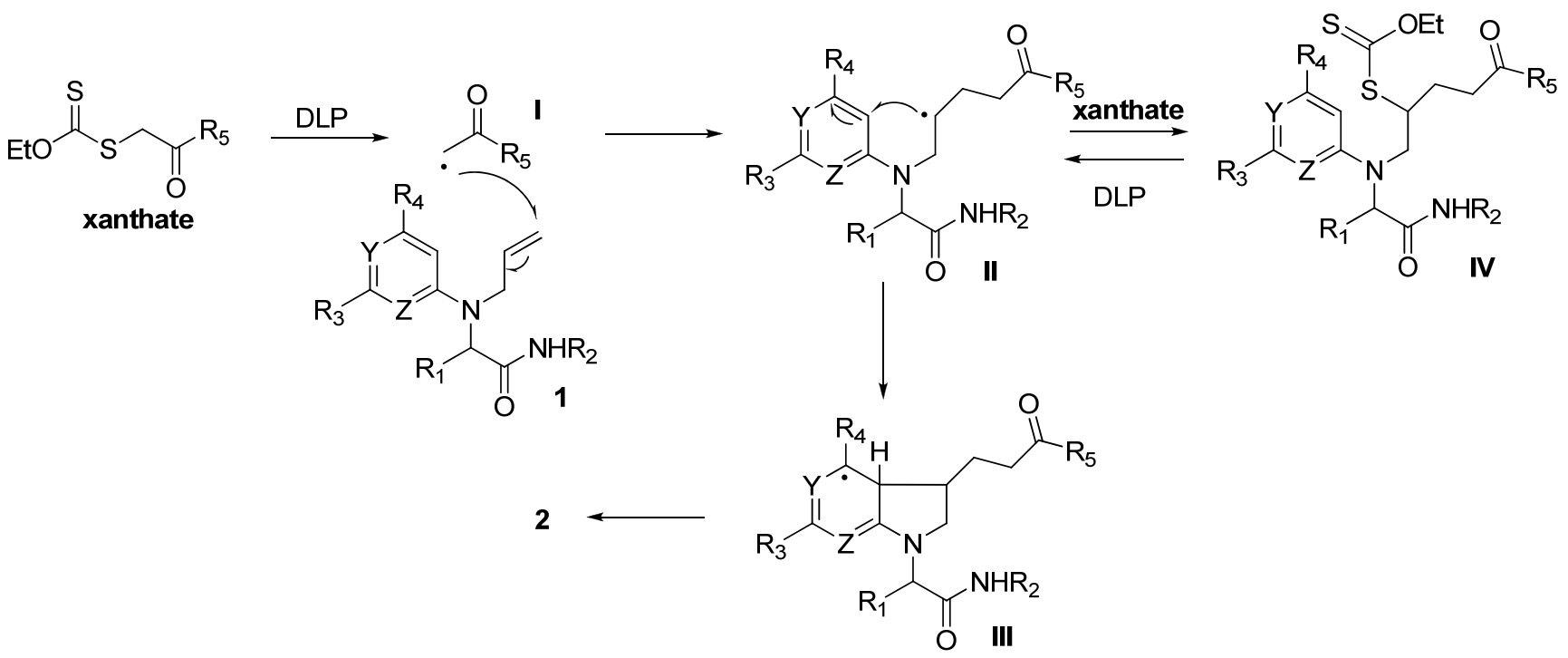

To evaluate the scope of this cyclization, the reaction was performed with the Ugi-Smiles adducts formerly prepared and the results are displayed in Table 2. 
Table 2. Radical additions-Cyclizations.

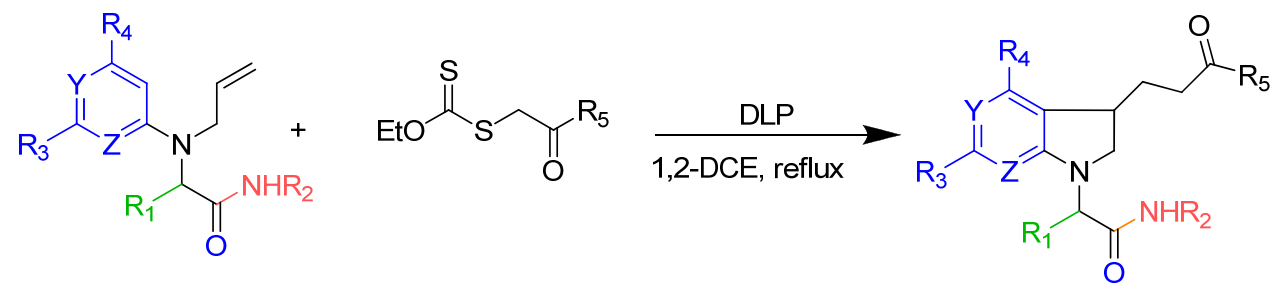

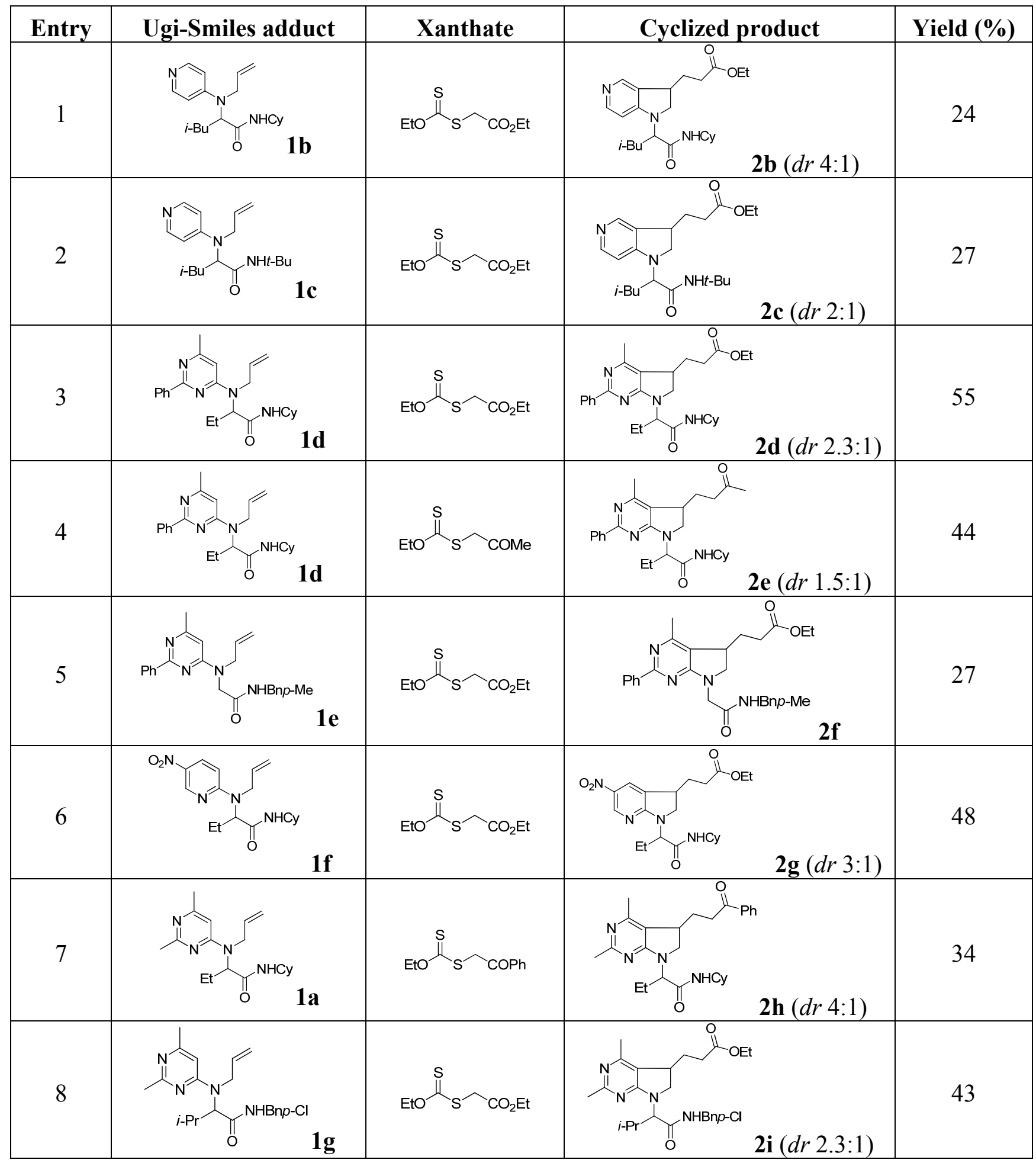


Pyridines and pyrimidines without any halogen substitutent at the 2 or 5 position can act as radical trapping agents in this sequence. In the study disclosed by Zard, initial attempts with 2-aminopyridine or 2-amino-5-methylpyridine as starting materials led to successful 1,2-addition product with the xanthate, but no indolizine could be formed in the following cyclization step [31]. Chloro or fluoro substituents are expected to lower the electron density in the aromatic ring leading to easier addition of the nucleophilic intermediate radical species, and this may explain the moderate yields observed. In our case, the reaction turns out to be poorly efficient with 4-aminopyridines, with yields not exceeding $30 \%$ (Table 2, Entries 1-2), a nitro group at the 5-position of the 2-aminopyridine ring activates the cyclization as a higher $48 \%$ isolated yield could be obtained (Table 2, Entry 6). Pyrimidines, in agreement with their higher electrophilicity, are better partners, giving yields over $50 \%$ with simple alkyl or aryl susbtituents on the heterocyclic core (Table 2, Entry 3).

\section{Experimental}

\subsection{General}

${ }^{1} \mathrm{H}-\mathrm{NMR}$ spectra were recorded on a $400 \mathrm{MHz}$ spectrometer (Bruker Avance 400), using $\mathrm{CDCl}_{3}$ solvent as reference and/or internal deuterium lock. ${ }^{13} \mathrm{C}$-NMR spectra were recorded on a $100.6 \mathrm{MHz}$ spectrometer (Bruker Avance 400). Two-dimensional NMR spectroscopy $\left[{ }^{1} \mathrm{H}-{ }^{1} \mathrm{H}\right.$ COSY spectra, ${ }^{1} \mathrm{H}-{ }^{13} \mathrm{C}$ COSY spectra (HSQC) and long-range ${ }^{1} \mathrm{H}-{ }^{13} \mathrm{C}$ COSY spectra (HMBC)], were carried out to determine the correlation between ${ }^{1} \mathrm{H}$ and ${ }^{13} \mathrm{C}$. The chemical shifts for all NMR spectra are expressed in parts per million to high frequency of TMS reference. Coupling constants $(J)$ are quoted in $\mathrm{Hz}$ and are recorded to the nearest $0.1 \mathrm{~Hz}$. The IR spectra were obtained using ATR accessories on a Bruker Tensor 27 instrument. High-resolution (HR) mass spectra were recorded on a Hewlett-Packard HP $5989 \mathrm{~B}$ spectrometer. TLC was carried out using precoated plates of silica gel 60F254 (SDS-Carlo-Erba).

\subsection{Ugi-Smiles Coupling Procedure}

To a $1 \mathrm{M}$ solution of hydroxyheteroaromatic compound in methanol were added successively 1.0 equivalent of amine, 1.0 equivalent of aldehyde and 1.0 equivalent of isocyanide. The resulting mixture was stirred at $65^{\circ} \mathrm{C}$ for $18-36 \mathrm{~h}$. The solvent was removed afterwards under reduced pressure to afford the Ugi-Smiles product after purification by flash chromatography on silica gel.

\subsection{DLP-Cyclisation Reaction Procedure}

A solution of Ugi-Smiles adduct $(1.0 \mathrm{mmol})$ and xanthate $(1.2 \mathrm{mmol})$ in 1,2-dichloroethane $(1.0 \mathrm{M})$ was refluxed for $5 \mathrm{~min}$ under argon before DLP was added $(60 \mathrm{~g}, 0.15 \mathrm{mmol})$ from the top of the condenser. Portions of DLP (60 mg, $0.15 \mathrm{mmol})$ were added every $20 \mathrm{~min}$ until complete disappearance of Ugi-Smile adduct and the starting xanthate, then the remaining DLP was added every 20 min until complete disappearance of the intermediate (TLC monitoring). Concentration under reduced pressure afforded an oily pale brown residue, which was purified by flash chromatography eluting with petroleum ether/diethyl ether to give the pure product as a mixture of two diastereomers. In all the cases, the spectral data are given for the major diastereomer. 
2-(N-Allyl-N-(2,6-dimethylpyrimidin-4-yl)amino)-N-cyclohexylbutanamide (1a). Yellow solid, (1.0 gm, 38\%), mp 124-125 ${ }^{\circ} \mathrm{C}$; ${ }^{1} \mathrm{H}-\mathrm{NMR}: \delta$ (ppm) 6.69 (br s, $\left.1 \mathrm{H}\right), 6.11(\mathrm{~s}, 1 \mathrm{H}), 5.86-5.69(\mathrm{~m}, 1 \mathrm{H}), 5.27-5.01$ $(\mathrm{m}, 3 \mathrm{H}), 4.08(\mathrm{dd}, 1 \mathrm{H}, J=4.8,17.0 \mathrm{~Hz}), 3.84(\mathrm{~d}, 1 \mathrm{H}, J=17.0 \mathrm{~Hz}), 3.75-3.63(\mathrm{~m}, 1 \mathrm{H}), 2.51(\mathrm{~s}, 3 \mathrm{H})$, $3.32(\mathrm{~s}, 3 \mathrm{H}), 2.13-2.00(\mathrm{~m}, 1 \mathrm{H}), 1.92-1.81(\mathrm{~m}, 1 \mathrm{H}), 1.78-1.59(\mathrm{~m}, 3 \mathrm{H}), 1.58-1.46(\mathrm{~m}, 2 \mathrm{H}), 1.40-1.22$ $(\mathrm{m}, 2 \mathrm{H}), 1.20-1.06(\mathrm{~m}, 2 \mathrm{H}), 1.02-0.93(\mathrm{~m}, 1 \mathrm{H}), 0.90(\mathrm{t}, 3 \mathrm{H}, J=7.3 \mathrm{~Hz}) ;{ }^{13} \mathrm{C}-\mathrm{NMR}: \delta(\mathrm{ppm}) 170.1$, 166.1, 165.3, 162.6, 133.5, 116.9, 100.3, 58.8, 47.6, 47.3, 32.9, 32.7, 26.1, 25.5, 24.5, 24.4, 24.3, 21.9, 10.9; IR (thin film): 2929, 2855, 2356, 1657, 1583, 1539, 1479, 1448, 1403, 1343, 1273, 1177, $1089 \mathrm{~cm}^{-1}$; HRMS: Calcd. for $\mathrm{C}_{19} \mathrm{H}_{30} \mathrm{~N}_{4} \mathrm{O}: 330.2420$, Found: 330.2425 .

2-(N-Allyl-N-(pyridin-4-yl)amino)- $N$-cyclohexyl-4-methylpentanamide (1b). Pale yellow solid, (475 mg, 69\%), mp 110-111 ${ }^{\circ} \mathrm{C},{ }^{1} \mathrm{H}-\mathrm{NMR}: \delta$ (ppm) 8.23 (dd, 2H, $\left.J=1.6,5.2 \mathrm{~Hz}\right), 6.59$ (dd, 2H, $J=1.6,5.2 \mathrm{~Hz}), 5.95-5.80(\mathrm{~m}, 2 \mathrm{H}), 5.29-5.21(\mathrm{~m}, 2 \mathrm{H}), 4.25(\mathrm{t}, 1 \mathrm{H}, J=6.9 \mathrm{~Hz}), 4.00(\mathrm{dd}, 1 \mathrm{H}, J=5.2$, $17.2 \mathrm{~Hz}), 3.90$ (dd, $1 \mathrm{H}, J=5.2,17.2 \mathrm{~Hz}), 3.80-3.69(\mathrm{~m}, 1 \mathrm{H}), 2.08-2.00(\mathrm{~m}, 1 \mathrm{H}), 1.86-1.76(\mathrm{~m}, 2 \mathrm{H})$, 1.70-1.53 (m, 5H), 1.39-1.25 (m, 2H), 1.15-1.00 (m, 3H), $0.92(\mathrm{~d}, 3 \mathrm{H}, J=6.7, \mathrm{~Hz}), 0.90(\mathrm{~d}, 3 \mathrm{H}$, $J=6.7 \mathrm{~Hz}$ ); ${ }^{13} \mathrm{C}-\mathrm{NMR}: \delta$ (ppm) 169.9, 153.0, 150.2, 133.2, 118.1, 108.3, 61.0, 49.7 , 48.4, 37.7, 32.9, 25.4, 25.2, 24.7, 22.9, 22.1; IR (thin film): 2930, 2853, 1652, 1596, 1544, 1513, 1450, 1235, $1169 \mathrm{~cm}^{-1}$; HRMS: Calcd. for $\mathrm{C}_{20} \mathrm{H}_{31} \mathrm{~N}_{3} \mathrm{O}: 329.2467$, Found: 329.2467.

2-(N-Allyl-N-(pyridin-4-yl)amino)-N-tert-butyl-4-methylpentanamide (1c). Pale yellow solid, (360 mg, 43\%), mp 111-112 ${ }^{\circ} \mathrm{C},{ }^{1} \mathrm{H}-\mathrm{NMR}: \delta(\mathrm{ppm}) 8.25$ (dd, 2H, $\left.J=1.6,5.0 \mathrm{~Hz}\right), 6.59$ (dd, 2H, $\left.J=1.6,5.0 \mathrm{~Hz}\right)$, $5.90-5.80(\mathrm{~m}, 2 \mathrm{H}), 5.28-5.19(\mathrm{~m}, 2 \mathrm{H}), 4.19(\mathrm{dd}, 1 \mathrm{H}, J=5.9,8.0 \mathrm{~Hz}), 4.00(\mathrm{ddt}, 1 \mathrm{H}, J=1.5,5.0$, $17.2 \mathrm{~Hz}), 3.89$ (ddt, $1 \mathrm{H}, J=1.5,5.0,17.2 \mathrm{~Hz}), 2.04-1.95(\mathrm{~m}, 1 \mathrm{H}), 1.65-1.53(\mathrm{~m}, 2 \mathrm{H}), 1.27(\mathrm{~s}, 9 \mathrm{H})$, 0.91 (d, 3H, $J=6.6 \mathrm{~Hz}), 0.89$ (d, 3H, $J=6.6 \mathrm{~Hz}) ;{ }^{13} \mathrm{C}-\mathrm{NMR}: \delta(\mathrm{ppm}) 170.0,153.0,149.8,133.1$, 117.9, 108.3, 61.4, 51.4, 49.6, 37.5, 28.5, 25.0, 22.8, 22.2; IR (thin film): 2964, 2930, 2871, 1676, 1596, 1544, 1513, 1454, 1367, 1266, 1231, $1172 \mathrm{~cm}^{-1}$; HRMS: Calcd. for $\mathrm{C}_{18} \mathrm{H}_{29} \mathrm{~N}_{3} \mathrm{O}$ : 303.2311, Found: 303.2309.

2-(N-Allyl-N-(6-methyl-2-phenylpyrimidin-4-yl)amino)-N-cyclohexylbutanamide (1d). white solid, (600 mg, 61\%), mp 114-115 ${ }^{\circ} \mathrm{C},{ }^{1} \mathrm{H}-\mathrm{NMR}: \delta$ (ppm) 8.50-8.27 (m, 2H), 7.60-7.38 (m, 3H), 6.60 (br s, $1 \mathrm{H}), 6.24(\mathrm{~s}, 1 \mathrm{H}), 5.94-5.76(\mathrm{~m}, 1 \mathrm{H}), 5.42-5.03(\mathrm{~m}, 3 \mathrm{H}), 4.11(\mathrm{~d}, 1 \mathrm{H}, J=17.0 \mathrm{~Hz}), 3.96(\mathrm{~d}, 1 \mathrm{H}$, $J=17.0 \mathrm{~Hz}), 3.77-3.61(\mathrm{~m}, 1 \mathrm{H}), 2.45(\mathrm{~s}, 3 \mathrm{H}), 2.24-2.08(\mathrm{~m}, 1 \mathrm{H}), 1.89-1.74(\mathrm{~m}, 2 \mathrm{H}), 1.68-1.36(\mathrm{~m}$, $4 \mathrm{H}), 1.33-1.13(\mathrm{~m}, 2 \mathrm{H}), 1.04-0.80(\mathrm{~m}, 6 \mathrm{H}) ;{ }^{13} \mathrm{C}-\mathrm{NMR}: \delta(\mathrm{ppm}) 170.2,166.0,162.8,162.6,138.2$, 133.5, 130.2, 128.4, 127.9, 117.1, 101.3, 59.7, 47.7, 32.8, 32.6, 25.3, 24.6, 24.4, 24.3, 21.6, 11.1; IR (thin film): 2929, 2850, 1658, 1591, 1570, 1526, 1474, 1443, 1377, 1260, 1208, 1181, $1024 \mathrm{~cm}^{-1}$; HRMS: Calcd. for $\mathrm{C}_{24} \mathrm{H}_{32} \mathrm{~N}_{4} \mathrm{O}$ : 392.2576, Found: 392.2575 .

2-(N-Allyl-N-(6-methyl-2-phenylpyrimidin-4-yl)amino)-N-p-tolylmethan-acetamide (1e). white solid, (498 mg, 60\%), mp 124-125 ${ }^{\circ} \mathrm{C},{ }^{1} \mathrm{H}-\mathrm{NMR}: \delta$ (ppm) 8.43-8.24 (m, 2H), 7.52-7.35 (m, 3H), $7.02(\mathrm{~d}$, $2 \mathrm{H}, J=8.0 \mathrm{~Hz}), 6.94(\mathrm{~d}, 2 \mathrm{H}, J=8.0 \mathrm{~Hz}), 6.79$ (br s, 1H), $6.25(\mathrm{~s}, 1 \mathrm{H}), 5.91-5.80(\mathrm{~m}, 1 \mathrm{H}), 5.31-5.14$ $(\mathrm{m}, 2 \mathrm{H}), 4.39(\mathrm{~s}, 2 \mathrm{H}), 4.29(\mathrm{~s}, 2 \mathrm{H}), 4.16(\mathrm{~s}, 2 \mathrm{H}), 2.44(\mathrm{~s}, 3 \mathrm{H}), 2.25(\mathrm{~s}, 3 \mathrm{H}) ;{ }^{13} \mathrm{C}-\mathrm{NMR}: \delta(\mathrm{ppm}) 169.5$, $166.4,163.3,162.1,137.9,136.9,134.7,131.3,130.2,129.2,128.2,128.0,127.3,117.8,99.9,52.2$, 51.4, 42.9, 24.5, 21.0; IR (thin film): 2920, 2356, 1653, 1591, 1570, 1531, 1496, 1439, 1408, 1374, 1260, 1230, 1194, 1068, $1024 \mathrm{~cm}^{-1}$; HRMS: Calcd. for $\mathrm{C}_{24} \mathrm{H}_{26} \mathrm{~N}_{4} \mathrm{O}: 386.2107$, Found: 386.2104 . 
2-(N-Allyl-N-(5-nitropyridin-2-yl)amino)-N-cyclohexylbutanamide (1f). Yellow solid, (508 mg, 41\%), mp 111-112 ${ }^{\circ} \mathrm{C},{ }^{1} \mathrm{H}-\mathrm{NMR}: \delta(\mathrm{ppm}) 9.04(\mathrm{~d}, 1 \mathrm{H}, J=2.2 \mathrm{~Hz}), 8.21(\mathrm{dd}, 1 \mathrm{H}, J=2.2,9.4 \mathrm{~Hz}), 6.52(\mathrm{~d}$, $1 \mathrm{H}, J=9.4 \mathrm{~Hz}), 6.18$ (br s, 1H), 5.87-5.72 (m, 1H), 5.38-5.11 (m, 3H), $4.23(\mathrm{dd}, 1 \mathrm{H}, J=4.8,17.0 \mathrm{~Hz})$, $4.03(\mathrm{~d}, 1 \mathrm{H}, J=17.0 \mathrm{~Hz}), 3.78-3.66(\mathrm{~m}, 1 \mathrm{H}), 2.19-2.03(\mathrm{~m}, 1 \mathrm{H}), 1.96-1.83(\mathrm{~m}, 1 \mathrm{H}), 1.80-1.49$ (m, 5H), $1.43-1.22(\mathrm{~m}, 2 \mathrm{H}), 1.21-1.06(\mathrm{~m}, 2 \mathrm{H}), 1.05-0.88(\mathrm{~m}, 4 \mathrm{H}) ;{ }^{13} \mathrm{C}-\mathrm{NMR}: \delta(\mathrm{ppm}) 169.2,160.7$, 145.6, 132.9, 132.7, 126.7, 117.6, 106.7, 60.0, 48.0, 47.9, 32.9, 32.8, 25.4, 24.5, 21.9, 10.8; IR (thin film): 2924, 2850, 1657, 1588, 1570, 1496, 1413, 1325, 1286, 1251, $111 \mathrm{~cm}^{-1}$; HRMS: Calcd. for $\mathrm{C}_{18} \mathrm{H}_{26} \mathrm{~N}_{4} \mathrm{O}_{3}$ : 346.2005, Found: 346.2016.

N-(4-Chlorobenzyl)-2-(N-allyl-N-(2,6-dimethylpyrimidin-4-yl)amino)-3-methylbutanamide (1g). White solid, (2.1 gm, 68\%), mp 97-98 ${ }^{\circ} \mathrm{C},{ }^{1} \mathrm{H}-\mathrm{NMR}: \delta(\mathrm{ppm}) 7.23(\mathrm{~d}, 2 \mathrm{H}, J=8.2 \mathrm{~Hz}), 7.07(\mathrm{~d}, 2 \mathrm{H}$, $J=8.2 \mathrm{~Hz}), 6.13(\mathrm{~s}, 1 \mathrm{H}), 5.70-5.55(\mathrm{~m}, 1 \mathrm{H}), 5.13(\mathrm{~d}, 2 \mathrm{H}, J=13.3 \mathrm{~Hz}), 4.91(\mathrm{br} \mathrm{s}, 1 \mathrm{H}), 4.40-4.25(\mathrm{~m}$, 2H), 4.10-3.90 (m, 2H), 2.56-2.42 (m, 1H), $2.38(\mathrm{~s}, 3 \mathrm{H}), 2.33(\mathrm{~s}, 3 \mathrm{H}), 1.02(\mathrm{~d}, 3 \mathrm{H}, J=6.4 \mathrm{~Hz})$, $0.81\left(\mathrm{~d}, 3 \mathrm{H}, J=6.4 \mathrm{~Hz}\right.$ ); ${ }^{13} \mathrm{C}-\mathrm{NMR}: \delta$ (ppm) 170.9, 166.1, 165.4, 162.6, 136.7, 133.1, 133.0, 128.9, 128.7, 117.4, 100.6, 42.6, 26.5, 26.1, 24.1, 19.9, 19.1; IR (thin film): 2968, 1671, 1583, 1535, 1474, 1403, 1339, 1268, 1203, $1085 \mathrm{~cm}^{-1}$; HRMS: Calcd. for $\mathrm{C}_{21} \mathrm{H}_{27} \mathrm{ClN}_{4} \mathrm{O}: 386.1873$, Found: 386.1868 .

Ethyl 3-(7-(1-(cyclohexylcarbamoyl)propyl)-6,7-dihydro-2,4-dimethyl-5H-pyrrolo[2,3-d]pyrimidin-5yl)propanoate (2a). Yellow liquid, (200 mg, 48\%), ${ }^{1} \mathrm{H}-\mathrm{NMR}: \delta(\mathrm{ppm}) 6.43(\mathrm{~d}, 1 \mathrm{H}, J=7.5 \mathrm{~Hz}), 4.40$ (t, $1 \mathrm{H}, J=7.7 \mathrm{~Hz}), 4.06(\mathrm{q}, 2 \mathrm{H}, J=6.8 \mathrm{~Hz}), 3.80-3.63(\mathrm{~m}, 2 \mathrm{H}), 3.40-3.25(\mathrm{~m}, 2 \mathrm{H}), 2.45(\mathrm{~s}, 3 \mathrm{H})$, 2.34-2.20 (m, 5H), 2.10-1.95 (m, 2H), 1.90-1.70 (m, 4H), 1.69-1.46 (m, 3H), 1.40-1.05 (m, 8H), 0.88 (t, 3H, $\left.J=7.3 \mathrm{~Hz}) ;{ }^{13} \mathrm{C}-\mathrm{NMR}: \delta(\mathrm{ppm}) 172.8\right), 169.1,166.3,166.2,157.2,116.4,60.6,57.9,50.5$, $47.8,35.0,32.9,32.8,30.4,28.9,25.8,25.4,24.5,24.4,20.8,20.5,14.2,10.7$; IR (thin film): 2929, 2855, 2356, 1731, 1657, 1609, 1570, 1517, 1448, 1400, 1312, 1277, 1260, 1168, 1168, 1089, $1028 \mathrm{~cm}^{-1}$; HRMS: Calcd. for $\mathrm{C}_{23} \mathrm{H}_{36} \mathrm{~N}_{4} \mathrm{O}_{3}: 416.2787$, Found: 416.2767 .

Ethyl 3-(1-(1-(cyclohexylcarbamoyl)-3-methylbutyl)-2,3-dihydro-1H-pyrrolo[3,2-c]pyridin-3-yl)propanoate (2b). Pale yellow liquid, ( $100 \mathrm{mg}, 24 \%),{ }^{1} \mathrm{H}-\mathrm{NMR}$ : $\delta(\mathrm{ppm}) 8.21(\mathrm{~s}, 1 \mathrm{H}), 8.12(\mathrm{~d}, 1 \mathrm{H}$, $J=5.8 \mathrm{~Hz}), 6.55$ (d, 1H, $J=5.8 \mathrm{~Hz}), 6.04(\mathrm{br} \mathrm{s}, 1 \mathrm{H}), 4.10$ (q, 2H, $J=7.1 \mathrm{~Hz}), 3.93(\mathrm{t}, 1 \mathrm{H}, J=6.5 \mathrm{~Hz})$, 3.80-3.60 (m, 3H), 3.00-2.88 (m, 1H), $2.29(\mathrm{t}, 2 \mathrm{H}, J=7.0 \mathrm{~Hz}), 2.02(\mathrm{dd}, 1 \mathrm{H}, J=7.0,13.4 \mathrm{~Hz})$, $1.95-1.78(\mathrm{~m}, 4 \mathrm{H}), 1.74-1.50(\mathrm{~m}, 6 \mathrm{H}), 1.35-1.15(\mathrm{~m}, 11 \mathrm{H}), 0.95-0.82(\mathrm{~m}, 2 \mathrm{H}) ;{ }^{13} \mathrm{C}-\mathrm{NMR}: \delta(\mathrm{ppm})$ $173.0,171.1,148.1,147.6,146.1,127.0,107.0,60.4,60.2,49.3,48.2,39.6,33.7,33.0,32.8,30.3$, 30.0, 29.0, 25.3, 24.8, 24.7, 24.5, 22.2, 14.1; IR (thin film): 2924, 2840, 2361, 1727, 1644, 1660, 1517, 1448, 1374, 1277, 1260, 1163, 1098, 1064, $1028 \mathrm{~cm}^{-1}$; HRMS: Calcd. for $\mathrm{C}_{24} \mathrm{H}_{37} \mathrm{~N}_{3} \mathrm{O}_{3}: 415.2835$, Found: 415.2837.

Ethyl 3-(1-(1-(tert-butylcarbamoyl)-3-methylbutyl)-2,3-dihydro-1H-pyrrolo[3,2-c]pyridin-3-yl)propanoate (2c). Pale brown liquid, $(85 \mathrm{mg}, 27 \%),{ }^{1} \mathrm{H}-\mathrm{NMR}: \delta(\mathrm{ppm}) 8.23(\mathrm{~s}, 1 \mathrm{H}), 8.14(\mathrm{~d}, 1 \mathrm{H}$, $J=5.8 \mathrm{~Hz}), 6.55(\mathrm{~d}, 1 \mathrm{H}, J=5.8 \mathrm{~Hz}), 5.85(\mathrm{br} \mathrm{s}, 1 \mathrm{H}), 4.10$ (q, 2H, $J=7.1 \mathrm{~Hz}), 3.82(\mathrm{t}, 1 \mathrm{H}, J=6.5 \mathrm{~Hz})$, 3.74-3.62 (m, 2H), 3.00-2.87 (m, 1H), $2.30(\mathrm{t}, 2 \mathrm{H}, J=7.0 \mathrm{~Hz}), 2.26-2.17(\mathrm{~m}, 1 \mathrm{H}), 2.06-196(\mathrm{~m}, 1 \mathrm{H})$, 1.94-1.83 (m, 1H), 1.65-1.55 (m, 2H), $1.32(\mathrm{~s}, 9 \mathrm{H}), 1.31-1.18(\mathrm{~m}, 9 \mathrm{H}) ;{ }^{13} \mathrm{C}-\mathrm{NMR}: \delta(\mathrm{ppm}) 173.0$, $171.1,156.2$, 148.0, 146.7, 127.4, 107.0, 60.9, 60.4, 51.2, 49.4, 39.6, 33.8, 33.3, 30.3, 28.5, 24.6, 22.2, 
14.2; IR (thin film): 2960, 2933, 2361, 1727, 1666, 1596, 1543, 1505, 1448, 1365, 1277, 1255, 1220, $1163 \mathrm{~cm}^{-1}$; HRMS: Calcd. for $\mathrm{C}_{22} \mathrm{H}_{35} \mathrm{~N}_{3} \mathrm{O}_{3}: 389.2678$, Found: 389.2679 .

Ethyl 3-(7-(1-(cyclohexylcarbamoyl)propyl)-6,7-dihydro-4-methyl-2-phenyl-5H-pyrrolo[2,3-d]pyrimidin-5-yl)propanoate (2d). Pale yellow liquid, (265 mg, 55\%), ${ }^{1} \mathrm{H}-\mathrm{NMR}: \delta(\mathrm{ppm}) 8.40-8.30(\mathrm{~m}$, 2H), 7.54-7.38 (m, 3H), 6.52 (br s, 1H), 4.63-4.45 (m, 1H), 4.09 (q, 2H, $J=7.0 \mathrm{~Hz}), 3.80-3.60$ (m, $2 \mathrm{H}), 3.50-3.25(\mathrm{~m}, 2 \mathrm{H}), 2.40(\mathrm{~s}, 3 \mathrm{H}), 2.37-2.24(\mathrm{~m}, 2 \mathrm{H}), 2.18-1.96(\mathrm{~m}, 2 \mathrm{H}), 1.94-1.70(\mathrm{~m}, 4 \mathrm{H})$, $1.63-1.38(\mathrm{~m}, 3 \mathrm{H}), 1.34-1.14(\mathrm{~m}, 6 \mathrm{H}), 1.05-0.80(\mathrm{~m}, 5 \mathrm{H}) ;{ }^{13} \mathrm{C}-\mathrm{NMR}: \delta(\mathrm{ppm}) 172.8,169.3,166.6$, $163.0,157.9,138.2,130.0,128.2,127.7,117.5,60.6,58.2,50.7,47.9,35.2,32.8,32.7,30.4,28.8$, 25.3, 24.4, 24.2, 21.0, 20.9, 14.2, 10.8; IR (thin film): 2972, 2929, 2850, 1731, 1662, 1605, 1562, 1531, 1452, 1377, 1312, 1246, $1163 \mathrm{~cm}^{-1}$, HRMS: Calcd. for $\mathrm{C}_{28} \mathrm{H}_{38} \mathrm{~N}_{4} \mathrm{O}_{3}$ : 478.2944, Found: 478.2950 .

N-Cyclohexyl-2-(5,6-dihydro-4-methyl-5-(3-oxobutyl)-2-phenylpyrrolo[2,3-d]pyrimidin-7-yl)butan-amide (2e). Yellow liquid, (75 mg, 44\%), ${ }^{1} \mathrm{H}-\mathrm{NMR}: \delta(\mathrm{ppm})$ 8.40-8.25 (m, 2H), 7.55-7.35 (m, 3H), $6.50(\mathrm{~d}$, $1 \mathrm{H}, J=8.0 \mathrm{~Hz}), 4.50(\mathrm{t}, 1 \mathrm{H}, J=6.6 \mathrm{~Hz}), 3.78-3.58(\mathrm{~m}, 2 \mathrm{H}), 3.45-3.25(\mathrm{~m}, 2 \mathrm{H}), 2.47(\mathrm{t}, 2 \mathrm{H}, J=7.5 \mathrm{~Hz})$, $2.40(\mathrm{~s}, 3 \mathrm{H}), 2.16-2.00(\mathrm{~m}, 5 \mathrm{H}), 1.90-1.66(\mathrm{~m}, 4 \mathrm{H}), 1.60-1.34(\mathrm{~m}, 4 \mathrm{H}), 1.30-1.14(\mathrm{~m}, 2 \mathrm{H}), 1.10-0.85$ (m, 5H); ${ }^{13} \mathrm{C}-\mathrm{NMR}: \delta$ (ppm) 207.9, 169.3, 166.5, 162.9, 157.8, 138.1, 130.0, 128.2, 127.7, 117.7, 58.2, 51.2, 47.8, 39.4, 35.1, 32.7, 30.0, 27.4, 25.3, 24.4, 24.3, 21.1, 21.0, 10.8; IR (thin film): 2929, 2853, 1710, 1656, 1603, 1564, 1508, 1454, 1377, 1317, 1247, 1159, $1064 \mathrm{~cm}^{-1}$, HRMS: Calcd. for $\mathrm{C}_{27} \mathrm{H}_{36} \mathrm{~N}_{4} \mathrm{O}_{2}$ : 448.2838, Found: 448.2836 .

Ethyl 3-(7-(N-p-tolylmethan-carbamoylmethyl)-6,7-dihydro-4-methyl-2-phenyl-5H-pyrrolo[2,3-d]pyrimidin-5-yl)propanoate (2f). Pale yellow liquid, (125 mg, 27\%), ${ }^{1} \mathrm{H}-\mathrm{NMR}: \delta(\mathrm{ppm}) 8.28(\mathrm{~d}, 2 \mathrm{H}$, $J=7.6 \mathrm{~Hz}), 7.45-7.34(\mathrm{~m}, 3 \mathrm{H}), 7.10(\mathrm{~d}, 2 \mathrm{H}, J=7.6 \mathrm{~Hz}), 7.00$ (d, 2H, $J=7.6 \mathrm{~Hz}), 6.95$ (br s, 1H), $4.50-4.25(\mathrm{~m}, 3 \mathrm{H}), 4.08-3.95(\mathrm{~m}, 3 \mathrm{H}), 3.75-3.65(\mathrm{~m}, 1 \mathrm{H}), 3.45-3.35(\mathrm{~m}, 2 \mathrm{H}), 2.40(\mathrm{~s}, 3 \mathrm{H}), 2.33-2.20$ (m, 5H), 2.10-2.00 (m, 1H), 1.96-1.85 (m, 1H), $1.20(\mathrm{t}, 3 \mathrm{H}, J=7.0 \mathrm{~Hz}) ;{ }^{13} \mathrm{C}-\mathrm{NMR}: \delta(\mathrm{ppm}) 173.0$, 169.0, 167.0, 163.6, 158.5, 138.0, 137.1, 135.0, 130.1, 129.4, 128.3, 128.0, 127.6, 117.4, 60.8, 55.8, 49.5, 43.2, 35.8, 30.8, 28.7, 21.1, 21.0, 14.2; IR (thin film): 2924, 2356, 1731, 1662, 1605, 1583, 1570, 1518, 1448, 1377, 1325, 1260, 1172, $1024 \mathrm{~cm}^{-1}$, HRMS: Calcd. for $\mathrm{C}_{28} \mathrm{H}_{32} \mathrm{~N}_{4} \mathrm{O}_{3}$ : 472.2474, Found : 472.2473 .

Ethyl 3-(1-(1-(cyclohexylcarbamoyl)propyl)-2,3-dihydro-5-nitro-1H-pyrrolo[2,3-b]pyridin-3-yl)propanoate (2g). Pale brown liquid, (297 mg, 48\%), ${ }^{1} \mathrm{H}-\mathrm{NMR}$ : $\delta$ (ppm) $8.86(\mathrm{~s}, 1 \mathrm{H}), 7.92$ (s, 1H), 6.04 (br s, 1H), 4.05 (t, 1H, $J=7.5 \mathrm{~Hz}), 4.15$ (q, 2H, $J=7.2 \mathrm{~Hz}), 4.00-3.83(\mathrm{~m}, 1 \mathrm{H}), 3.79-3.62$ (m, $1 \mathrm{H}), 3.55-3.30(\mathrm{~m}, 2 \mathrm{H}), 2.44-2.30(\mathrm{~m}, 2 \mathrm{H}), 2.20-1.98(\mathrm{~m}, 2 \mathrm{H}), 1.94-1.76(\mathrm{~m}, 3 \mathrm{H}), 1.74-1.64(\mathrm{~m}$, $3 \mathrm{H}), 1.62-1.50(\mathrm{~m}, 2 \mathrm{H}), 1.34-1.25(\mathrm{~m}, 6 \mathrm{H}), 1.20-1.10(\mathrm{~m}, 1 \mathrm{H}), 0.95(\mathrm{t}, 3 \mathrm{H}, J=7.2 \mathrm{~Hz}){ }^{13} \mathrm{C}-\mathrm{NMR}: \delta$ (ppm) 172.5, 168.1, 164.6, 146.7, 136.6, 126.7, 125.7, 60.8, 58.5, 51.9, 48.2, 36.2, 33.0, 31.0, 30.9, 29.5, 25.4, 24.6, 24.5, 21.4, 14.3, 10.7; I.R. (thin film): 2933, 2850, 2356, 1731, 1657, 1609, 1574, 1517, 1496, 1443, 1377, 1291, 1186, 1090, $1024 \mathrm{~cm}^{-1}$, HRMS: Calcd. for $\mathrm{C}_{22} \mathrm{H}_{32} \mathrm{~N}_{4} \mathrm{O}_{5}: 432.2373$, Found: 432.2386 . 
N-Cyclohexyl-2-(5,6-dihydro-2,4-dimethyl-5-(3-oxo-3-phenylpropyl)pyrrolo[2,3-d]pyrimidin-7-yl)butanamide (2h). Pale brown liquid, (150 mg, 34\%), ${ }^{1} \mathrm{H}-\mathrm{NMR}: \delta(\mathrm{ppm}) 7.90$ (d, 2H, $\left.J=7.8 \mathrm{~Hz}\right)$, $7.58-7.54(\mathrm{~m}, 1 \mathrm{H}), 7.48-7.41(\mathrm{~m}, 2 \mathrm{H}), 6.48(\mathrm{~d}, 1 \mathrm{H}, J=7.8 \mathrm{~Hz}), 4.41(\mathrm{t}, 1 \mathrm{H}, J=7.7 \mathrm{~Hz}), 3.80-3.65$ (m, 2H), 3.46-3.30 (m, 2H), 3.08-2.83 (m, 2H), 2.45 (s, 3H), 2.30 (s, 3H), 2.26-2.14 (m, 1H), 2.08-1.96 (m, 1H), 1.94-1.40 (m, 7H), 1.30-0.98 (m, 5H), 0.90 (t, 3H, $J=7.3 \mathrm{~Hz}) ;{ }^{13} \mathrm{C}-\mathrm{NMR}: \delta(\mathrm{ppm}) 199.3$, 169.2, 166.3, 166.1, 157.2, 136.6, 133.2, 128.6, 127.9, 116.8, 58.0, 50.7, 47.9, 35.2, 34.1, 33.0, 32.8, 28.2, 25.8, 25.4, 24.4, 21.1, 20.6, 10.8; IR (thin film): 2929, 2853, 1671, 1611, 1568, 1515, 1446, 1404, 1358, 1317, 1270, 1259, 1202, $1083 \mathrm{~cm}^{-1}$, HRMS: Calcd. for $\mathrm{C}_{27} \mathrm{H}_{36} \mathrm{~N}_{4} \mathrm{O}_{2}$ : 448.2838, Found: 448.2843.

Ethyl 3-(7-(1-(4-chlorobenzylcarbamoyl)-2-methylpropyl)-6,7-dihydro-2,4-dimethyl-5H-pyrrolo[2,3d]pyrimidin-5-yl)propanoate (2i). Pale yellow liquid, (203 mg, 43\%), ${ }^{1} \mathrm{H}-\mathrm{NMR}: \delta(\mathrm{ppm}) 7.22(\mathrm{~d}, 2 \mathrm{H}$, $J=8.0 \mathrm{~Hz}), 7.07(\mathrm{~d}, 2 \mathrm{H}, J=8.0 \mathrm{~Hz}), 6.97(\mathrm{~d}, 1 \mathrm{H}, J=6.0 \mathrm{~Hz}), 4.39(\mathrm{dd}, 2 \mathrm{H}, J=6.0,14.7 \mathrm{~Hz}), 4.12(\mathrm{q}$, $2 \mathrm{H}, J=7.0 \mathrm{~Hz}), 4.04-3.97(\mathrm{~m}, 1 \mathrm{H}), 3.73(\mathrm{t}, 1 \mathrm{H}, J=7.0 \mathrm{~Hz}), 3.40-3.20(\mathrm{~m}, 2 \mathrm{H}), 2.51-2.38(\mathrm{~m}, 1 \mathrm{H})$, $2.35(\mathrm{~s}, 3 \mathrm{H}), 2.28(\mathrm{~s}, 3 \mathrm{H}), 2.23(\mathrm{t}, 2 \mathrm{H}, J=7.5 \mathrm{~Hz}), 2.10-1.90(\mathrm{~m}, 1 \mathrm{H}), 1.80-1.65(\mathrm{~m}, 1 \mathrm{H}), 1.25(\mathrm{t}, 3 \mathrm{H}$, $J=7.0 \mathrm{~Hz}), 1.00$ (d, 3H, $J=7.0 \mathrm{~Hz}), 0.87$ (d, 3H, $J=7.0 \mathrm{~Hz}) ;{ }^{13} \mathrm{C}-\mathrm{NMR}: \delta(\mathrm{ppm}) 172.8,169.6,166.1$, $165.9,157.3,136.7,133.2,128.9,128.7,116.6,64.0,60.6,51.5,42.6,35.2,30.6,28.9,26.4,25.7$, 20.6, 19.5, 19.1, 14.2; IR (thin film): 2964, 2924, 1731, 1671, 1609, 1570, 1531, 1469, 1403, 1273, 1177, 1094, $1011 \mathrm{~cm}^{-1}$, HRMS: Calcd. for $\mathrm{C}_{25} \mathrm{H}_{33} \mathrm{ClN}_{4} \mathrm{O}_{3}$ : 472.2241, Found : 472.2244.

\section{Conclusions}

The reported two-step cascade constitutes a concise entry into pyrrolidino- pyridines and pyrimidines. Compared with previous results obtained on xanthate cascades performed on $N$-allylamino- pyridine and pyrimidines, these new couplings significantly widen the scope of these reactions. The modest efficiency of the whole process is counter-balanced by the simple experimental procedure and the straightforward access to important biologically relevant scaffolds [40-43]. This work is a new example of the potential of radical chemistry in Ugi post-condensations.

\section{Acknowledgements}

P. P. thanks the ANR-CP2D Program (ANR MUSE) for a fellowship.

\section{References and Notes}

1. Dömling, A. Recent Developments in Isocyanide Based Multicomponent Reactions in Applied Chemistry. Chem. Rev. 2006, 106, 17-89 and references cited therein.

2. Dömling, A.; Ugi, I. Multicomponent reactions with isocyanides. Angew. Chem. Int. Ed. 2000, 39, 3168-3210.

3. Lu, K.; Luo, T.; Xiang, Z.; You, Z.; Fathi, R.; Chen, J.; Yang, Z. A Concise and DiversityOriented Strategy for the Synthesis of Benzofurans and Indoles via Ugi and Diels-Alder Reactions. J. Comb. Chem. 2005, 7, 958-967.

4. Paulvannan, K. An Atom-Economical approach to Conformationally Constrained Tricyclic Nitrogen Heterocycles via Sequential and tandem Ugi/Intramolecular Diels-Alder Reaction of Pyrrole. J. Org. Chem. 2004, 69, 1207-1214. 
5. Akritopoulou-Zanze, I.; Djuric, S.W. Synthesis of novel fused isoxazoles and isoxazolines by sequential Ugi/INOC reactions. Tetrahedron Lett. 2004, 45, 3421-3423.

6. Akritopoulou-Zanze, I.; Gracias, V.; Djuric, S.W. A versatile synthesis of fused triazolo derivatives by sequential Ugi/alkyne-azide cycloaddition reactions. Tetrahedron Lett. 2004, 45, 8439-8441.

7. Akritopoulou-Zanze, I.; Whitehead, A.; Waters, J.E.; Henry, R.F.; Djuric, S.W. Synthesis of Novel and Uniquely Shaped 3-Azabicyclo[4.2.0]octan-4-one Derivatives by Sequential Ugi/[2 + 2] Ene-Enone Photocycloadditions. Org. Lett. 2007, 9, 1299-1302.

8. Akritopoulou-Zanze, I.; Wang, Y.; Zhao, H.; Djuric, S.W. Synthesis of substituted fused pyridines, pyrazines and pyrimidines by sequential Ugi/inverse electron demand Diels-Alder transformations. Tetrahedron Lett. 2009, 50, 5773-5776.

9. Fayol, A.; Zhu, J. Multicomponent synthesis of epoxy-tetrahydronaphthyridine and structural diversification by subsequent fragmentation. Tetrahedron 2005, 61, 11511-11519.

10. Gracias, V.; Moore, J.D.; Djuric, S.W. Sequential Ugi/Heck cyclization strategies for the facile construction of highly functionalized N-heterocyclic scaffolds Tetrahedron Lett. 2004, 45, 417-420.

11. Xiang, Z.; Luo, T.; Lu, K.; Cui. J.; Shi, X.; Fathi, R.; Chen, J.; Yang, Z. Concise Synthesis of Isoquinoline via the Ugi and Heck Reactions. Org. Lett. 2004, 6, 3155-3158.

12. Krelaus, R.; Westermann, B. Preparation of peptide-like bicyclic lactams via a sequential Ugi reaction-olefin metathesis approach. Tetrahedron Lett. 2004, 45, 5987-5990.

13. Kazmaier, U.; Hebach, C.; Watzke, A.; Maeir, S.; Mues, H.; Huch, V. A straightforward approach towards cyclic peptides via ring-closing metathesis-scope and limitations. Org. Biomol. Chem. 2005, 3, 136-145.

14. Basso, A.; Banfi, L.; Riva, R.; Guanti, G. Preparation of optically pure fused polycyclic scaffolds by Ugi reaction followed by olefin and enyne metathesis. Tetrahedron 2006, 62, 8830-8837.

15. Kalinski, C.; Umkehrer, M.; Ross, G.; Kolb, J.; Burdack, C.; Hillerb, W. Highly substituted indol2-ones, quinoxalin-2-ones and benzodiazepin-2,5-diones via a new Ugi(4CR)-Pd assisted N-aryl amidation strategy. Tetrahedron Lett. 2006, 47, 3423-3426.

16. Marcaccini, S.; Miliciani, M.; Pepino, R. A facile synthesis of 1,4-benzodiazepine derivatives via Ugi four-component condensation .Tetrahedron Lett. 2005, 46, 711-713.

17. Nixey, T.; Tempest, P.; Hulme, C. Two-step solution-phase synthesis of novel quinoxalinones utilizing a UDC (Ugi/de-Boc/cyclize) strategy. Tetrahedron Lett. 2002, 43, 1637-1639.

18. Banfi, L.; Basso, A.; Casuscelli, F.; Guanti, G.; Naz, F.; Riva, R.; Zito, P. Synthesis of Novel Isochromene Derivatives by Tandem Ugi Reaction/Nucleophilic Substitution. Synlett 2010, 85-88.

19. Ignacio, J.M.; Macho, S.; Marcaccini, S.; Pepino, R.; Torroba, T. A Facile Synthesis of 1,3,5Trisubstituted Hydantoins via Ugi Four-Component Condensation. Synlett 2005, 2005, 3051-3054.

20. Xing, X.; Wu, J.; Luo, J.; Dai, W.-M. C-N Bond-Linked Conjugates of Dibenz $[b, f][1,4]$ oxazepines with 2-Oxindole. Synlett 2006, 2099-2103.

21. Nixey, T.; Kelly, M.; Semin, D.; Hulme, C. Short solution phase preparation of fused azepinetetrazoles via a UDC (Ugi/de-Boc/cyclize) strategy. Tetrahedron Lett. 2002, 43, 3681-3684.

22. El Kaim, L.; Grimaud, L.; Miranda, L.D.; Vieu, E. Ugi/xanthate cyclizations as a radical route to lactam scaffolds. Tetrahedron Lett. 2006, 47, 8259-8261. 
23. El Kaim, L.; Grimaud, L.; Miranda, L.D.; Vieu, E. From Simple Ugi Adducts to Indane and deltaAmidomalonate: New $\mathrm{Mn}(\mathrm{OAc})_{3}$ Induced Radical Cascades. Org. Lett. 2007, 9, 4171-4173.

24. Zamudio-Medina, A.; García-González, M.C.; Padilla, J.; González-Zamora, E. Synthesis of a tetracyclic lactam system of Nuevamine by four-component reaction and free radical cyclization. Tetrahedron Lett. 2010, 51, 4837-4839.

25. El Kaim, L.; Grimaud, L.; Miranda, L.D.; Vieu, E.; Cano-Herrera, M.-A.; Perez-Labrada, K. New xanthate-based radical cyclization onto alkynes. Chem. Commun. 2010, 46, 2489-2491.

26. Gámez-Montaño, R.; Ibarra-Rivera, T.; El Kaim, L.; Miranda, L.D. Efficient Synthesis of Azaspirodienones by Microwave-Assisted Spirocyclization of Xanthate-Containing Ugi Adducts. Synthesis 2010, 8, 1285-1290.

27. Yu, H.; Gai, T.; Sun, W.L.; Zhang, M.S. Radical reduction of Passerini 3CR adducts by $\mathrm{SmI}_{2}$ /HMPA. Chin. Chem. Lett. 2011, 22, 379-381.

28. Zard, S.Z. On the Trail of Xanthates: Some New Chemistry from an old Functional Group. Angew. Chem. Int. Ed. Engl. 1997, 36, 672-685.

29. Quiclet-Sire, B.; Zard, S.Z. The Degenerative Radical Transfer of Xanthates and Related Derivatives: An Unusually Powerful Tool for the Creation of Carbon-Carbon Bonds. Top. Curr. Chem. 2006, 264, 201-236.

30. Ly, T.-L.; Quiclet-Sire, B.; Sortais, B.; Zard, S.Z. A Convergent Approach to Indolines and Indanes. Tetrahedron Lett. 1999, 40, 2533-2536.

31. Bacqué, E.; El Qacemi, M.; Zard, S.Z. Tin-Free Radical Cyclizations for the Synthesis of 7Azaoxindoles, 7-Azaindolines, Tetrahydro[1,8]naphthyridines, and Tetrahydro-5H-pyrido[2,3b]azepin-8-ones. Org. Lett. 2004, 6, 3671-3674.

32. El Qacemi, M.; Ricard, L.; Zard, S.Z. An unprecedented radical ring closure on a pyridine nitrogen. Chem. Commun. 2006, 4422-4424.

33. Laot, Y.; Petit, L.; Zard, S.Z. Unusual radical addition on a heteroaromatic nitrogen. A convenient access to new pyrimidine derivatives. Chem. Commun. 2010, 46, 5784-5786.

34. Laot, Y.; Petit, L.; Zard, S.Z. Synthesis of Fluoroazaindolines by an Uncommon Radical ipso Substitution of a Carbon-Fluorine Bond. Org. Lett. 2010, 12, 3426-3429.

35. Laot, Y.; Petit, L.; Diem My Tran, N.; Zard, S.Z. Fluoroazaindolines by an Uncommon Radical ipso-Substitution of a Carbon-Fluorine Bond. Aust. J. Chem. 2011, 64, 416-425.

36. El Kaim, L.; Grimaud, L.; Oble, J. Phenol Ugi-Smiles Systems: Strategies for the Multicomponent N-Arylation of Primary Amines with Isocyanides, Aldehydes, and Phenols. Angew. Chem. Int. Ed. 2005, 44, 7961-7964.

37. El Kaim, L.; Gizolme, M.; Grimaud, L.; Oble, J. Smiles Rearrangements in Ugi- and PasseriniType Couplings: New Multicomponent Access to $O$ - and $N$-Arylamides. J. Org. Chem. 2007, 72, 4169-4180.

38. El Kaim, L.; Gizolme, M.; Grimaud, L.; Oble, J. Direct Access to Heterocyclic Scaffolds by New Multicomponent Ugi-Smiles Couplings. Org. Lett. 2006, 8, 4019-4021.

39. The structure of the major diastereomers could not be determined with usual NMR techniques.

40. Aso, K.; Kobayashi, K.; Mochizuki, M.; Kanzaki, N.; Sako, Y.; Yano, T. Discovery of pyrrolo[2,3-d]pyrimidin-4-ones as corticotropin-releasing factor 1 receptor antagonists with a carbonyl-based hydrogen bonding acceptor. Bioorg. Med. Chem. Lett. 2011, 21, 2365-2371. 
41. Mueller, C.E.; Geis, U.; Grahner, B.; Lanzner, W.; Eger, K. Chiral Pyrrolo[2,3-d]pyrimidine and Pyrimido[4,5-b]indole Derivatives: Structure-Activity Relationships of Potent, Highly Stereoselective $A_{1}$-Adenosine Receptor Antagonists. J. Med. Chem. 1996, 39, 2482-2491.

42. Aso, K.; Hitaka, T.; Yukishige, K.; Ootsu, K.; Akimoto, H. Synthesis and Antitumor Activity of Pyrrolo[2,3-d]pyrimidine Antifolates with a Bridge Chain Containing a Nitrogen Atom. Chem. Pharm. Bull. 1995, 43, 256-261.

43. Simard, D.; Leblanc, Y.; Berthelette, C.; Zaghdane, M.H.; Molinaro, C.; Wang, Z.; Gallant, M.; Lau, S.; Thao, T.; Hamel, M.; Stocco, R.; Sawyer, N.; Sillaots, S.; Gervais, F.; Houle, R.; Levesque, J.-F. Azaindoles as potent CRTH2 receptor antagonists. Bioorg. Med. Chem. Lett. 2011, 21, 841-845.

Sample Availability: Not available.

(C) 2011 by the authors; licensee MDPI, Basel, Switzerland. This article is an open access article distributed under the terms and conditions of the Creative Commons Attribution license (http://creativecommons.org/licenses/by/3.0/). 